\title{
Professional Well-being Work Party of WFSA: It is Time to Think and Take Action Regarding the Occupational Health of Anesthesiologists
}

The nature and intensity of the work performed by anesthesiologists have changed dramatically in recent decades. The advent of new technologies has expanded the surgical horizon, allowing intervention in more challenging medical conditions. The pressure of a growing economic competitiveness and the need to do more with a reduced workforce are associated with the emergence of more difficult cases. This transformation impacted occupational well-being of anesthesiologists. Occupational well-being is a reflex of work satisfaction, leading to overall improvement in quality of life. Finding a healthy solution to integrate the work into our life in order to provide personal balance and satisfaction will result in greater general well-being.

Current epidemiological studies on occupational health of physicians are focused primarily on the investigation and analysis of somatic and/or psychological pathologies, such as degenerative, cardiovascular, toxic, and infectious pathologies, fatigue and nervous breakdown, depression, and chemical dependency ${ }^{1,2}$. On the other hand, it is evident how little has been dome regarding the prevention of these adverse occupational problems and the continuous maintenance of occupational well-being of physicians. The need for prevention is increased by the growing risks related to occupational health of anesthesiologists, as is common knowledge, a vulnerable group.
Recently, on the area of occupational health of anesthesiologists, the knowledge about the risks of somatic and/or psychological pathologies, worsened by the stress of clinical practice, has improved the diagnosis, prevention, and management of these adverse conditions ${ }^{3,4}$. However, it is very important that anesthesiologists be aware of the aspects of their work that is causing more stress and they should know how better working conditions can be implemented to maintain their occupational health. The need for such improvements is more evident when one considers whether support systems for anesthesiologists with any deficiency have been established by professional defense associations, the state, or governmental organizations.

\section{AN EXAMPLE OFF A WELL-BEING SUPPORT SYSTEM}

A careful analysis of information regarding the occupational health of physicians, especially anesthesiologists, leads to a very disturbing, and even alarming, conclusion, that such support systems are virtually non-existent. Information from a support system admittedly competent, the Canadian Physician Health Services, in 2002, can be a guide: the Physician Health Program of OMA (Ontario Medical Association) is a confidential Canadian service that provides assistance

\section{Problem Types for New Cases Ontario Phisician Health Program}

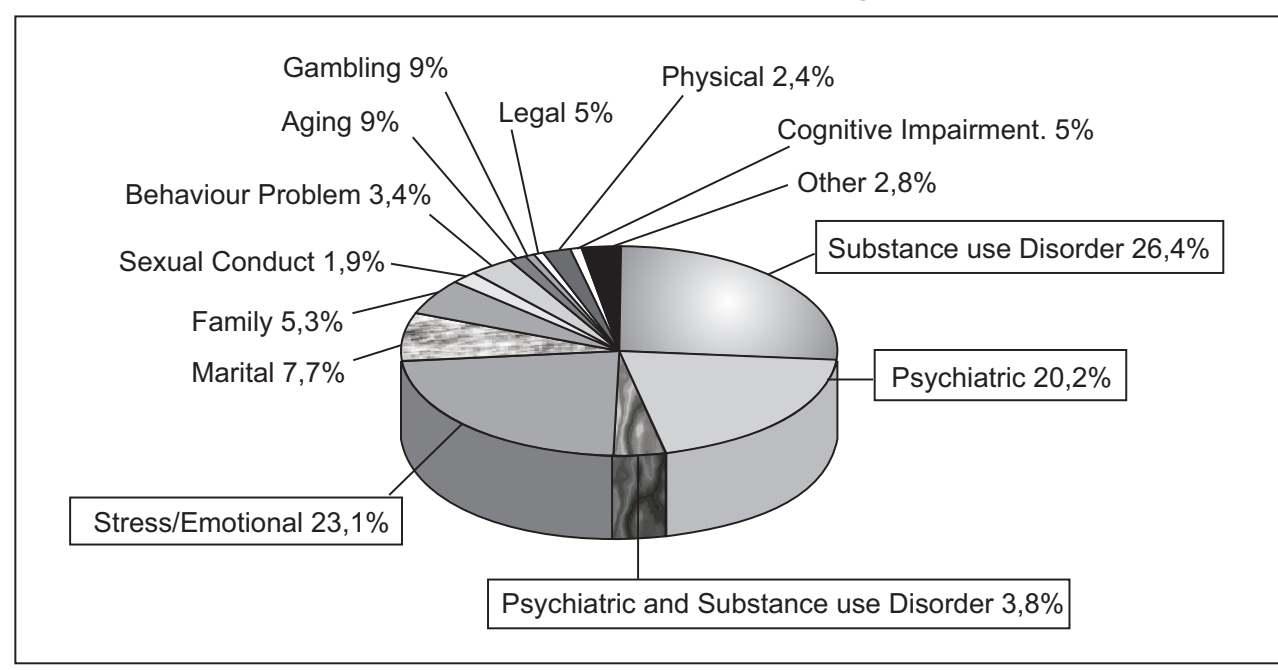

Figure 1 - Casuistry of the Ontario Physician Health Program. 
to the physician who is afflicted by occupational pathologies (Figure 1). The Centre of the Canadian Medical Association for Physician Health and Well-being, an institution that granted more credibility and efficacy to activities and claims of the Ontario Medical Association, was created in 2000.

Epidemiological data documented by the Ontario Medical Association, showed an increase in the number of psychopathological diseases related to medical practice when compared to purely somatic pathologies resulting from problems like infections, irradiation, contamination, and gas inhalation.

Based on the attention given to occupational health and well-being of Canadian physicians, Dr. Michael Myers, a professor of clinical Psychiatry at the University of British Columbia, published a book, by the Canadian Medical Association, warning against the risk factors of occupational pathologies, raising money for their diagnosis, treatment, and support in Canada ${ }^{5}$. Undoubtedly, this is an initiative that should be followed by other medical associations.

\section{SPECIFIC RISKS}

Current characteristics inherent to the clinical practice of anesthesiologists may result in specific correlations with psychological pathologies. Risks of acute and chronic fatigue and high levels of occupational stress should be observed during clinical practice, as well as in resident training programs.

Recently, Professor Oli Meretoja published an article titled "We should work less at night" ${ }^{6}$. He concluded:

"There is increasingly more evidence that performance of physicians is poorer if they work excessive prolonged shifts or at night. These work patterns decrease the quality of care and increase medical expenses. Furthermore, night workers have serious health risks due to their non-physiologic work shifts. Effective ways of reducing overall consequences of fatigue and night work include minimizing the amount of work performed at night and establishing rules regarding the maximum working hours for each work shift." 6

In Brazil, the department related to the approach of chemically dependent physicians (Uniad) of the Escola de Medicina de São Paulo recently presented a series, shown in Table I, including 57 anesthesiologists with clinical evidence of drug addiction. Opioids (53\%), benzodiazepines (30\%), and alcohol $(23 \%)$ were the drugs used more often. Chemical dependency, especially opioids, significantly increase the difficulty of giving support and effective rehabilitation treatment, especially due to the high risk of relapse and the risk of suicide or death secondary to overdose. Another rehabilitation difficulty facing anesthesiologists who are dependent on opioids is the relatively greater availability and easy access to drugs in the operating room, recovery rooms, and postoperative care units.

Table II shows the frequency of psychiatric comorbidities associated with chemical dependency among anesthesiologists. As mentioned before, there is a relationship between
Table I - Casuistry of UNIAD - UNIFESP (São Paulo), Chemical Dependent Physician Treatment Center (UNIAD - UNIFESP)

\begin{tabular}{|c|c|c|c|}
\hline \multicolumn{4}{|c|}{ (Drugs used more often) } \\
\hline Drugs & $\begin{array}{l}\text { Total } \\
\text { n (\%) }\end{array}$ & $\begin{array}{l}\text { Alarming use } \\
\mathrm{n}(\%)\end{array}$ & $\begin{array}{l}\text { Dependency } \\
\text { n (\%) }\end{array}$ \\
\hline Alcohol & $20(35.1)$ & $7(12.3)$ & $12(22.8)$ \\
\hline Benzodiazepines & $20(35.1)$ & $3(5.2)$ & $17(29.8)$ \\
\hline Opioids & $34(59.6)$ & $4(7.0)$ & $30(52.6)$ \\
\hline Cocaine and Crack & $3(5.2)$ & $3(5.2)$ & $0(0)$ \\
\hline Marijuana & $6(10.5)$ & $4(7.0)$ & $2(3.5)$ \\
\hline Amphetamines & 6 (10.5) & $2(3.5)$ & $4(7.0)$ \\
\hline Inhalants & $1(1.8)$ & $1(1.8)$ & $0(0)$ \\
\hline
\end{tabular}

Table II - Prevalence of Comorbidities in Chemically Dependent Individuals at UNIAD - UNIFESP (São Paulo)

\begin{tabular}{lll}
\hline \multicolumn{2}{l}{ Diagnosis of Psychological Pathologies (ICD 10) } \\
& $\mathbf{n}$ & $\%$ \\
\hline Total cases of comorbidities & 24 & 42.1 \\
Depression (F32 and F33) & 12 & 21.0 \\
Personality Disorders (F60) & 6 & 10.5 \\
Bipolar Disorders (F31) & 5 & 8.7 \\
Anxiety Disorders (F41) & 4 & 7.0 \\
Schizophrenia (F20) & 1 & 1.7 \\
\hline
\end{tabular}

the psychogenic pathologies developed by anesthesiologists (fatigue, depression, nervous breakdown etc.) and the chemical dependency syndrome. During their training, physicians should be alerted regarding the risks of death due to drug addiction. This is valid especially for residents and trainees who present an elevated risk of developing chemical dependency. Recently, Collins et al. analyzed American anesthesiology residents during 10 years and concluded that $70 \%$ of residents with chemical dependency were able to resume the practice of medicine after a successful treatment program ${ }^{7}$. However, only $60 \%$ of those who returned to medicine were able to successfully continue in anesthesiology, and $9 \%$ had premature death. The authors concluded that the anesthesiology resident with chemical dependency can obtain better results if he chooses a less risky specialty.

Professor Francis Bonnet and colleagues published a national study on the incidence of drug dependency among French anesthesiologists ${ }^{8}$. They demonstrated that $11 \%$ of anesthesiologists who answered the questionnaire were users or dependents of one or more drugs, except tobacco. Alcohol (59\% of the cases) and anti-anxiety/hypnotic drugs (41\%) were the substances used more often. The incidence of drug addiction increased with age. Dependent individuals mentioned problems at work that might have contributed to the development of their pathology.

The Burnout Syndrome is a well defined medical condition characterized by emotional exhaustion, depersonalization, and reduced personal accomplishments ${ }^{~}$. Emotional exhaustion of an individual, especially due to excessive working demands, personal conflicts in interpersonal relationships, as well as performance of professional duties, is considered the 
Table III - Conclusions of the "Study of Relationships among Occupational Stress Level and the Work Conditions: In Anesthesiology Training Programs in Brazil"

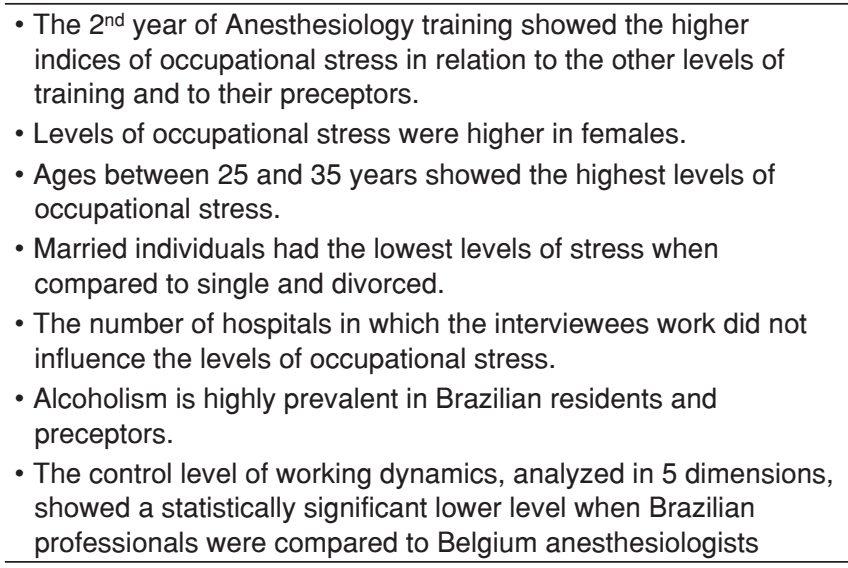

initial step of this syndrome. Depersonalization is characterized by emotional insensitivity of the health professional. The presence of this symptom is essential for the diagnosis of Burnout Syndrome, whereas other symptoms can be found in general case of depression. And the feeling of reduced personal achievements (lack of ability) reveals a negative self-assessment associated with a work-related lack of satisfaction and sadness.

Professor De Keyser and her working group, constituted of psychologists and anesthesiologists of the Université de Liège, Belgium, observed a high incidence of Burnout Syndrome among Belgian anesthesiologists, especially young professional under 30 years of age ${ }^{10}$. There is a special concern regarding young residents involved in clinical-educational training, where the working load and occupational stress usually are excessive, sometimes due to their lack of clinical experience. Unfortunately, some trainees exchange the recreational use of potentially addictive drugs by abuse of substances that cause chemical dependency as a way of dealing with difficult situations. Eventually, the chemical dependency of the health care professional can jeopardize the safety of the surgical patient.

Fatigue and sleep deprivation are other factors that can also reduce the safety of patients. There is increasing evidence that excessive work reduces safety and increased the incidence of mistakes by tired anesthesiologists when compared to those that are rested ${ }^{11-14}$.

Concerns about the occupational health of physicians increased in the last two decades. The first works on the matter were published by the BMJ and Western $\mathrm{J}$ Med. These studies emphasized that up to $46 \%$ of all Canadian physicians presented advanced stages of the Burnout Syndrome ${ }^{15}$.

Currently, without a doubt, there is an exacerbated tendency for the development of somatic and psychogenic pathologies with an occupational etiology. Possibilities of developing actions to protect the mental and physical health of health professionals have been presented. An option would be the creation of systems that support the health of anesthesiologists, establishing institutional and governmental policies to prevent occupational diseases. This argument can be simplified in the title of a BMJ editorial, written by Dr. Gavin Yamey: "We should move away from a disease model and focus on positive function as a means of prophylaxis (Occupational Well-being)" 16.

The Brazilian Society of Anesthesiology (SBA, from the Portuguese) has shown an increased interest regarding occupational health of anesthesiologists since 2000. Its function is to understand, alert, and influence this type of situation, which has a significant importance on the life of anesthesiologists. Actions developed have been supported by the Occupational Health Committee of this entity and by the World Federation of Societies of Anesthesiologists (WFSA), through its "Professional Well-being Work Party" (PWWP/WFSA). The Committee of Occupational Health of the SBA conducted an epidemiologic study, in association with Professor Isabelle Hansez, of the Department of Work Psychology of Université of Liège, in Belgium, whose objective was to evaluate the occupational stress level and the degree of adaptability to working conditions of residents and their preceptors in the Anesthesiology Training Programs connected to the SBA and to the Ministry of Education. The main results are summarized in Table III (not published).

On the spring 2010, the PWWP of WFSA conducted an investigation involving 120 member-societies, using a questionnaire, whose objective was to identify the incidence of occupational health problems among the members of a specific society and the approaches used by those societies to treat the occupational health of anesthesiologists.

Results showed that more than $90 \%$ of National Societies consider the Burnout Syndrome as a problem among their members, but only $14 \%$ developed some sort of strategy to face it. The PWWP of WFSA organized a special symposium on the topic for the next World Congress of Anesthesiologists, in 2012 in Buenos Aires.

Jenny Firth-Cozens, special counselor of modernization of postgraduate courses, in the BMJ editorial Doctors, their wellbeing, and their stress. It is time to be pro-active regarding stress - and to prevent it summarizes our feelings regarding the attitude in relation to the occupational well-being of anesthesiologists ${ }^{17}$. She ends her text with the following phrase: "Stress is here to stay, and the sooner we accept that fighting it is natural, besides being an essential part of patient safety, the faster the lives of doctors and their patients will improve."

Organizations involved with structuring the education and/ or medical practice need to understand that the consequences of occupational health problems of physicians and residents not only result in worrisome changes in somatic and psychological health of anesthesiologists, but they also hinder the safety of medical practices regarding anesthesiologists and patients, and, at the same time, they increase the costs of medical assistance. 
To conclude, we need to be more aggressive in formatting medical education regarding the occupational health risks of physicians, specifically anesthesiologists, which can harm their health and well-being. Moreover, it has been well documented that these risks to anesthesiologists can represent serious consequences for patient safety. National policies to prevent and handle the burnout syndrome and related pathologies in health care professional also must be developed.
Gastão F. Duval Neto (Brasil)

President of PWWP / WFSA

Member of the Executive Committee of WFSA

Members of PWWP

Francis Bonet (France)

Steve Howard (USA)

Pratyush Gupta (India)

Olli Meretoja (Finland)

Roger Moore (USA)

Max-André Doppia (France) 